Pacific Journal of Mathematic

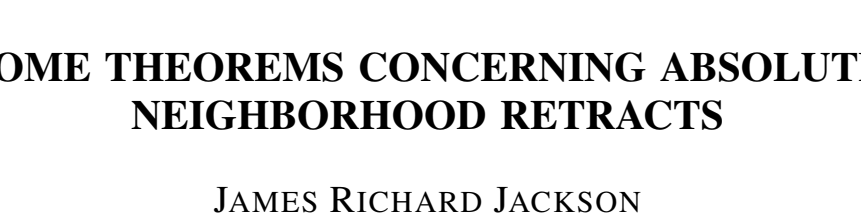




\title{
SOME THEOREMS CONCERNING ABSOLUTE NEIGHBORHOOD RETRACTS
}

\author{
JAMES R. JACKSON
}

1. Introduction. If $X$ is a subset of a topological space $X^{*}$, and there exists a mapping (continuous function) $\rho: X^{*} \rightarrow X$ such that $\rho(x)=x$ for $x \in X$, then $X$ is called a retract of $X^{*}$, and $\rho$ is called a retraction. If $U$ is a neighborhood of $X$ in $X^{*}$ (that is, a set open in $X^{*}$ and containing $X$ ), and there exists a retraction $\rho^{\prime}: U \longrightarrow X$, then $X$ is called a neighborhood retract of $X^{*}$. If $X$ is a separable metric space such that every homeomorphic image of $X$ as a closed subset of a separable metric space $M$ is a neighborhood retract of $M$, then $X$ is called an absolute neighborhood retract or an $A N R$. It is with such spaces that we shall be principally concerned. They are of particular interest because of their usefulness in homotopy theory, and also because every locally-finite polyhedron is an $A N R$ [5].

Section 2 is concerned with two theorems of point-set topological nature. For many other theorems along this line, see $[3, \S 10]$.

In $\S 3$, we are concerned with spaces of mappings into $A N R$ 's, and with homotopy problems involving $A N R$ 's; in particular, we obtain certain restrictions on the cardinality of collections of homotopy classes of mappings into ANR's . For closely related results, see [4], especially the corollary to Theorem 5.

2. Theorems in point-set-topology. The following theorem is a slight generalization of a standard result.

THEOREM 1. If a subset $X_{0}$ of an ANR $X$ is a neighborhood retract of $X$, then $X_{0}$ is an $A N R$.

This generalization consists in not requiring that $X_{0}$ be closed. For the more restricted theorem and its proof (which in actuality establishes Theorem 1), see $[3, \S 10.1]$.

An interesting corollary of Theorem 1 is that an open subset of an ANR is an ANR.

Received June 26, 1951. Parts of this paper are included in the author's thesis, University of California, Los Angeles, 1952.

Pacific J. Math. 2(1952), 185-189 
We now prove a theorem which shows that the study of $A N R$ 's may-for many purposes-be reduced to the study of connected $A N R$ 's.

THEOREM 2. A topological space $X$ is an ANR if and only if $X$ has at most countably many components, each of which is open in $X$, and each of which is itself an ANR.

Proof of Theorem 2. Suppose $X$ is an ANR. Then it is well known that $X$ is locally connected, and hence has open components. Since $X$ must be perfectly separable, this implies that there are at most countably many components; and that they are themselves $A N R$ 's follows from the corollary to Theorem 1 . This proves the necessity.

Now suppose $X=\cup C_{i}$, where each $C_{i}$ is an open component of $X$, and also an $A N R$, and suppose that $X$ is a closed subset of a separable metric space $M$. For definiteness we assume that the set of $C_{i}$ 's is denumerably infinite; the proof must be simplified slightly if the union is finite.

Since $C_{1}$ and $\left(C_{2} \cup C_{3} \cup \ldots\right)$ are disjoint closed subsets of $M$, we can pick open subsets $U_{1}$ and $U_{1}^{*}$ of $M$ with $C_{1} \subset U_{1}$ and $\left(C_{2} \cup C_{3} \cup \ldots\right) \subset U_{1}^{*}$, and with $U_{1} \cap U_{1}^{*}=\phi$ (the null set). Then obviously $U_{1} \cap\left(C_{2} \cup C_{3} \cup \ldots\right)=\phi$ (where $\bar{U}_{1}$ is the closure of $U_{1}$ ).

Having picked $U_{1}, U_{2}, \ldots, U_{n-1}$ such that each $U_{i}$ is open, contains $C_{i}$, is disjoint from the other $U_{i}$, and has a closure disjoint from $C_{j}$ for all $j \neq i$; we see that the sets

$$
\bigcup_{1}^{n-1} \bar{U}_{i} \cup \underset{n+1}{\bigcup} C_{i} \equiv K_{n}
$$

and $C_{n}$ are disjoint closed sets. Hence we may choose disjoint open sets $U_{n} \supset C_{n}$ and $U_{n}^{*} \supset K_{n}$.

Then we easily see that by induction we may pick open sets $U_{i}(i=1,2, \ldots)$ so that for each $i$ we have $C_{i} \subset U_{i}$, and so that for $i \neq j$ we have $U_{i} \cap U_{j}=\phi$.

Since each $C_{i}$ is a closed $A N R$ subset of $M$, we may pick open sets $V_{i}(i=1$, $2, \ldots)$ such that each $V_{i}$ contains $C_{i}$, and retractions $\rho_{i}: V_{i} \rightarrow C_{i}, i=1$, $2, \cdots$

Define

$$
\rho: \bigcup_{1}^{\infty}\left(U_{i} \cap V_{i}\right) \rightarrow X
$$

by setting $\rho(x)=\rho_{i}(x)$ for $x \in U_{i} \cap V_{i}$. It is clear that $\rho$ retracts a neighborhood of $X$ onto $X$, as required.

3. Results in homotopy theory. Our next theorem has a corollary concerning homotopy groups and the homotopy classification problem. 
THEOREM 3 : Let $X$ be a compact topological space, and let $Y$ be an ANR. Then the space $Y^{X}$ of continuous functions on $X$ into $Y$ has open, arcwise-connected components.

If $X_{0} \subset X$, and $y_{0} \in Y$, then the space $Y^{X}\left\{X_{0}, y_{0}\right\}$ of functions in $Y^{X}$ which carry $X_{0}$ to $y_{0}$ also has open, arcwise-connected components.

(We give the function spaces the topology of uniform convergence, which for compact $X$ 's coincides with the more general compact-open topology.)

In the case that $X$ is metric, a known theorem tells us that $Y^{X}$ is itself an $A N R$, whence the first result follows easily. However, the second result is of perhaps greater interest, because of its bearing on homotopy groups (see below). We shall prove the second result; the proof of the first proceeds similarly, but is slightly easier.

Proof of Theorem 3. Let $d$ be a metric on $Y$. The topology on our function space is obtained by taking

$$
d^{*}(f, g)=\sup _{x \in X} d(f(x), g(x))
$$

as a metric for functions $f$ and $g$. We first show that if $f \in Y^{X}\left(X_{0}, y_{0}\right)$ then there exists an $\epsilon>0$ such that if $d^{*}(f, g)<\epsilon$, then $g$ can be joined to $f$ by an arc in $Y^{X}\left\{X_{0}, y_{0}\right\}$.

By Wojdyslawski's imbedding theorem, we may take $Y$ to be a closed subset of a convex subset $Z$ of a Banach space $S$. Since $Y$ is an $A N R$, there exists a retraction $\rho: U \longrightarrow Y$ of a neighborhood $U$ of $Y$ in $Z$ onto $Y$. Since $f(X)$ is compact and disjoint from the closed subset $Z-U$ of $Z$, we may choose $\epsilon>0$ such that the $\epsilon$-neighborhood of $f(X)$ in $Z$ is contained in $U$.

Now suppose $d^{*}(f, g)<\epsilon$. Define

$$
H(x, t)=(1-t) f(x)+t g(x) \quad((t, x) \in X \times I) .
$$

It is clear that each $H(x, t) \in U$, so we may define

$$
h(x, t)=\rho(H(x, t)) \quad((t, x) \in X \times I) .
$$

Plainly $h: X \times I \rightarrow Y$ is continuous. Hence by a thenrem of R.H. Fox [2], the function $\rho^{*}: I \longrightarrow Y X$ defined by

$$
\rho^{*}(t)(x)=h(x, t) \quad(t \in I, x \in I) .
$$

is also continuous. One sees easily that $\rho^{*}(0)=f, \rho^{*}(1)=g$, and that the image of $\rho^{*}$ is actually in $Y^{X}\left\{X_{0}, y_{0}\right\}$. 
This proves the statement made at the beginning of the proof. This statement makes it obvious that the arc-components are open. One shows from this statement that the arc-components coincide with the components, in exactly the same way that one shows that a connected open subset of Euclidean space is arcwise connected.

A result of Fox [2] implies that if $X$ is either locally compact and regular, or locally separable (that is, satisfies the first axiom of countability), and the function spaces are given the compact-open topology, then the arcs in $Y^{X}$ and in $Y^{X}\left\{X_{0}, y_{0}\right\}$ are essentially identical with the homotopies of functions in these spaces. It follows from Theorem 3 and the equivalence of the topologies for a compact $X$ that if $X$ is compact and either regular or locally separable, and $Y$ is an $A N R$, then $Y^{X}$ and $Y^{X}\left\{X_{0}, y_{0}\right\}$ have open components, and these components coincide with the homotopy classes of the function spaces.

Arens [1] has given a theorem which - specialized to the cases with which we are dealing - states that if $X$ has a basis of cardinal number $a$, and $Y$ has a basis of cardinal number $b$, then $Y^{X}$ (and hence $Y^{X}\left\{X_{0}, y_{0}\right\}$ as well) has a basis whose cardinal number does not exceed both $a$ and $b$. Eliminating trivial cases by assuming that $X$ does not have finite basis, we see that each of our function spaces has a basis of cardinal number not greater than $a$. This easily implies that the collections of open components of our function spaces cannot have cardinalities greater than $a$; with the conclusion obtained by use of Fox's theorem, this establishes the following result.

THEOREM 4: Let the topological space $X$ be compact, and either regular or locally separable. Suppose that $X$ has a basis of (infinite) cardinal number a. Let $Y$ be an ANR. Then the collection of homotopy classes of functions in $Y^{X}$, and also the collection of homotopy classes of functions in $Y^{X}\left\{X_{0}, y_{0}\right\}$, each has cardinality not greater than a.

The following theorem is an immediate corollary to the second conclusion of of the above theorem.

THEOREM 5 : A homotopy group of an ANR is at most countable.

The necessity for strong hypotheses in Theorems 3, 4, and 5 is indicated by examples we have found which show that $(i)$ an arcwise-connected, locally-connected, and compact subset of the plane may have an uncountable fundamental group; (ii) the homotopy classes of the space of mappings of the 1-sphere into an arcwise-connected and locally-connected subset of 3-space need not coincide with the components of this function space; (iii) the homotopy classes of the space of mappings of a separable metric, arcwise-connected, locally-contractible, compact 
space into itself need not be open in this space of mappings.

\section{REFERENCES}

1. R. F. Arens, A topology for spaces of transformations, Ann. of Math. 47 (1946), 480-495.

2. R. H. Fox, On topologies for function spaces, Bull. Amer. Math. Soc. 51 (1945), 429-432.

3. S. T. Hu, Homotopy theory (v. 1), Dittoed typescript prepared by the Department of Mathematics, Tulane University, 1950.

4. C. Kuratowski, Sur les espaces localement connexes et péanien en dimension n, Fund. Math. 24 (1935), 269-287.

5. S. D. Liao, On non-compact absolute neighborhood retracts, Academy Sinica Science Record 2 (1949), 249-262.

University of California, Los Angeles 



\section{PACIFIC JOURNAL OF MATHEMATICS}

\section{EDITORS}

R. M. RoBINSON

University of California Berkeley 4, California

\section{*R. P. Dilworth}

Califomia Institute of Technology Pasadena 4, California

\section{E. F. BECKENBACH, Managing Editor}

University of California

Los Angeles 24, California

*During the absence of Herbert Busemann in 1952.

\section{ASSOCIATE EDITORS}

\author{
R. P. DILWORTH \\ HERBERT FEDERER \\ MARSHALL HALL
}

\author{
P. R. HALMOS \\ HEINZ HOPF
}

R. D. JAMES

\author{
BØRGE JESSEN \\ PAUL LÉVY \\ GEORGE PÓLYA
}

\author{
J. J. STOKER \\ E. G. STRAUS
}

KÖSAKU YOSIDA

\section{SPONSORS}

UNIVERSITY OF BRITISH COLUMBIA

CALIFORNIA INSTITUTE OF TECHNOLOGY

UNIVERSITY OF CALIFORNIA, BERKELEY

UNIVERSITY OF CALIFORNIA, DAVIS

UNIVERSITY OF CALIFORNIA, LOS ANGELES

UNIVERSITY OF CALIFORNIA, SANTA BARBARA

OREGON STATE COLLEGE

UNIVERSITY OF OREGON

\author{
UNIVERSITY OF SOUTHERN CALIFORNIA \\ STANFORD UNIVERSITY \\ WASHINGTON STATE COLLEGE \\ UNIVERSITY OF WASHINGTON \\ AMERICAN MATHEMATICAL SOCIETY \\ NATIONAL BUREAU OF STANDARDS, \\ INSTITUTE FOR NUMERICAL ANALYSIS
}

Mathematical papers intended for publication in the Pacific Journal of Miathematics should be typewritten (double spaced), and the author should keep a complete copy. Manuscripts may be sent to any of the editors. All other communications to the editors should be addressed to the managing editor, E. F. Beckenbach, at the address given above.

Authors are entitled to receive 100 free reprints of their published papers and may obtain additional copies at cost.

The Pacific Journal of Mathematics is published quarterly, in March, June, September, and December, by the University of California, Berkeley 4, California. The price per volume (4 numbers) is $\$ 8.00$; single issues, $\$ 2.50$. Special price to individual faculty members of supporting institutions and to individual members of the American Mathematical Society: $\$ 4.00$ per volume; single issues, $\$ 1.25$.

Subscriptions, orders for back numbers, and changes of address should be sent to the publishers, University of California Press, Berkeley 4, California.

Printed at Ann Arbor, Michigan. Entered as second class matter at the Post Office, Berkeley, California.

\section{UNIVERSITY OF CALIFORNIA PRESS • BERKELEY AND LOS ANGELES}




\section{Pacific Journal of Mathematics}

\section{Vol. 2, No. $2 \quad$ February, 1952}

L. Carlitz, Some theorems on Bernoulli numbers of higher order...

Watson Bryan Fulks, On the boundary values of solutions of the heat equation........................................ 141

John W. Green, On the level surfaces of potentials of masses with fixed center of gravity................................... 147

Isidore Heller, Contributions to the theory of divergent series .......... 153

Melvin Henriksen, On the ideal structure of the ring of entire functions . . . 179

James Richard Jackson, Some theorems concerning absolute neighborhood retracts........................................ 185

Everett H. Larguier, Homology bases with applications to local connectedness ................................... 191

Janet McDonald, Davis's canonical pencils of lines ................ 209

J. D. Niblett, Some hypergeometric identities . . . . . . . . . . . . . . . . . . 219

Elmer Edwin Osborne, On matrices having the same characteristic equation...................................... 227

Robert Steinberg and Raymond Moos Redheffer, Analytic proof of the

Lindemann theorem ................................. 231

Edward Silverman, Set functions associated with Lebesgue area ......... 243

James G. Wendel, Left centralizers and isomorphisms of group algebras . . 251

Kosaku Yosida, On Brownian motion in a homogeneous Riemannian space. 